\title{
Nécessité et contingence chez Hegel et Spinoza
}

\section{Necessity and Contingency in Hegel and Spinoza}

RÉSUMÉ: Hegel et Spinoza font de la nécessité le centre de leur système. A ce titre, ils sont suspectés de méconnaître l'importance de la contingence. Le but de cet article est de montrer qu'ils ne négligent nullement le problème de la contingence et qu'ils l'articulent à la catégorie de nécessité. Partant de ce constat, l'auteur essaye de penser le potentiel de Hegel eu égard aux débats actuels sur le statut de la contingence en faisant contraster sa position avec celle de Spinoza.

MOTS CLÉS: HEGEL - SPINOZA - NÉCESSITÉ - CONTINGENCE - MÉTAPHYSIQUE
ABSTRACT: Hegel and Spinoza elaborate a system made of necessity. That is why they are both suspected to disregard contingency. The aim of this article is to show that they articulate necessity to contingency. Consequently they can put light on contemporary metaphysical debates on the status of contingency. Hegel is particularly interesting in regard to this question. Within his logic, the relation between necessity and contingency appears in a new light : the idea of a dialectical interconnection of modal categories.

KEY WORDS: HEGEL - SPINOZA - NECESSITY - CONTINGENCY - METAPHYSICS 
À la mémoire de Bernard Mabille

$\mathrm{E}$ 1893, BRADLEY faisait remarquer la chose suivante : «L'écrivain qui travaille dans le domaine de la métaphysique n’a pas la tâche aisée. Engagé dans un sujet qui, plus que d'autres, demande la paix de l'esprit, il se trouve impliqué dans une sorte de lutte [quant à la légitimité de sa démarche et de ses buts], avant même qu'il n'entre dans les controverses de son propre champ d'investigation. $»^{1}$ Les relations du sens commun à la métaphysique ne se sont guère améliorées depuis. Martial Guéroult note ainsi au début de ses leçons sur la philosophie de l'histoire de la philosophie : «Il fut un temps où l'on pouvait risquer de paraître prétentieux en parlant de métaphysique et de «système». Mais si la métaphysique et l'esprit de système étaient déjà bien dépréciés à la fin du XVIIIème siècle, à une époque où Kant lui-même hésitait à énoncer «le nom si décrié de métaphysique», ils sont tombés aujourd'hui en un tel point de discrédit, que celui qui voudrait les défendre risque moins d'être accusé de présomption que d'ignorance, de naïveté, ou de sottise.»²

La conséquence de ce déclin de la métaphysique classique ${ }^{3}$ est que l'on tente d'interprèter les auteurs classiques «sans métaphysique». Kervégan a essayé de définir ce type d'appréhension en ce qui concerne $\mathrm{Hegel}^{4}$, suscitant un large débat, dont témoigne la publication récente d'un colloque organisé en France «Hegel au présent. Une relève de la métaphysique ?» ${ }^{5}$.

L'objet de ma contribution n'est nullement de thématiser tous les aspects du problème, je voudrais juste clarifier et investiguer un aspect central de la suspicion envers la métaphysique. Cet aspect concerne l'idée de nécessité. A une époque qui prétend généralement relever de la postmétaphysique, tout semble marqué du sceau de la contingence. Parler de nécessité ne semble rien de plus qu'une prétention.

On peut toutefois se demander sur quoi repose l'opinion selon laquelle la nécessité ne serait qu'une simple prétention. D’une façon intéressante, Vittorio Hösle a montré que les partisans de la contingence devaient reconnaître la

[1] F.H. Bradley, Appearance and Reality. London : Allen and Unwin $1897^{2}$, p. 1.

[2] M. Gueroult, Philosophie de l'histoire de la philosophie. Paris : Aubier-Montaigne 1979 , p. 13.

[3] On assiste moins à une disparition pure et simple de la métaphysique qu’à sa transformation dans de nouvelles formes au rang desquelles on peut compter la métaphysique analytique ou encore le réalisme spéculatif.

[4] J-F. KeRVÉGAN, «Hegel sans métaphysique?», in : L'effectif et le rationnel. Hegel et l'esprit objectif. Paris : Vrin 2007, pp. 7-15.

[5] J-F. KeRvéGAN, B. Mabille (eds.), Hegel au présent. Une relève de la métaphysique?. Paris : CNRS-éditions, 2012. 
propre contingence de leur position ${ }^{6}$. Depuis l'argument de Hösle, de nouvelles idées ont néanmoins contribué à alimenter le débat et à le réorienter. Je pense ici au réalisme speculatif et, plus précisément, à l'idée défendue par Quentin Meillassoux selon laquelle il y aurait une nécessité de la contingence 7 . L'exigence d'explorer à nouveau frais le nexus entre nécessité et contingence s'impose donc. L'on tentera d'y répondre en méditant sur la pensée de Hegel et de Spinoza.

En commentant ces auteurs, il nous faut être extrêmement prudent. La première chose à faire est de distinguer une nécessité à l'œuvre dans le système chez Hegel et Spinoza d'une nécessité de ces systèmes. Hormis un Kojève qui semble indiquer que Hegel aurait nécessairement raison et qu'on ne pourrait faire que répéter son discours ${ }^{8}$, rares sont les penseurs pour qui le système hégélien ou le système spinoziste s'impose comme une nécessité d'un bout à l'autre. Si l'un ou l'autre de ces penseurs était absolument nécessaire à l'exercice de la pensée, on ne pourrait guère comprendre comment il y a eu des philosophes avant Hegel ou Spinoza. Bernard Mabille propose ici une intéressante distinction. Pour lui, le système hégélien n'est pas nécessaire, mais les catégories de pensée qu'il met en jeu sont, quant à elles, nécessaires pour construire un discours ayant prétention à la vérité. Il y aurait ainsi une nécessité dans la philosophie de Hegel, mais pas une nécessité de sa philosophie. ${ }^{9}$ Reste à comprendre le statut de cette nécessité.

Un moyen de l'appréhender est de penser la nécessité dans son positionnement à la modalité qui lui est contradictoire, en l'occurrence la catégorie de la contingence. En bonne logique aristotélicienne, on dira que les deux catégories s'excluent : on aurait pour une même classe d'objet une nécessité sans contingence ou une contingence sans nécessité.

Hors du carré logique, on peut toutefois distinguer, sans ici prétendre aucunement à l'exhaustivité, différents cas de figures suivant que l'on pense les catégories contradictoires en termes d'imbrication ou de dénotation mutuelle plutôt que d'exclusivité : une contingence de la nécessité, une contingence dans la nécessité, une nécessité de la contingence et une nécessité dans la contingence. Dans ce qui suit, on va analyser ces différents cas de figure à travers la philo-

[6] V. HösLE, «Begründungsfragen des objektiven Idealismus», in Philosophie und Begründung. Frankfurt: Suhrkamp 1987, pp. 212-267.

[7] Q. Meillassoux, "Après la finitude. Essai sur la nécessité de la contingence». Paris : Seuil 2006.

[8] Cf. A. Kojève, Le concept, le temps et le discours. Introduction au système du savoir. Paris : NRF 1990. Il est intéressant de noter que pour Hegel l'argument selon lequel on serait condamner à répéter son système était considéré comme poussif. (HEGEL, Écrits sur la religion (1822-29), trad. Georget. Paris : Vrin 2001, p. 152).

[9] Voir B. MABille, «La libération de la métaphysique» in : Ce peu d'espace autour. Six essais sur la métaphysique et ses limites. Chatou : La Transparence 2010, pp. 122-123. 
sophie de Hegel et de Spinoza. Notre choix s'est naturellement porté vers eux dans la mesure où faisant de la nécessité le maître mot de leur système ${ }^{10}$, ils apparaissent aux yeux de maints contemporains comme les exemples rêvés de métaphysiciens aveugles à la contingence du monde ${ }^{11}$; Hegel aurait ainsi fait du réel quelque chose d'entièrement rationnel ${ }^{12}$.

\section{Le statut des modalités aléthiques chez Hegel et Spinoza}

Hegel et Spinoza ont tous deux été compris comme des penseurs réduisant le monde à un système de nécessité. Nous voudrions montrer que, chez eux et contrairement aux idées reçues, le fait de penser la nécessité n'implique pas d'ignorer la contingence. Le statut accordé à la contingence est toutefois radicalement différent chez les deux philosophes et exprime exemplairement la divergence entre leurs systèmes philosophiques. Tant l'un que l'autre envisagent à divers endroits de leurs oeuvres l'ensemble des modalités aléthiques traditionnelles (nécessité, contingence, possibilité, impossibilité). Hegel les voit comme des catégories co-constitutives de l'effectivité (Wirklichkeit). Il montre ainsi dans la doctrine de l'essence ${ }^{13}$ que la nécessité logique se doit d'affronter la contingence du monde pour se rendre effective. La contingence est alors reconnue, comme quelque chose dont il faut faire l'épreuve pour concevoir la nécessité sous un mode effectif. Chez Spinoza, la contingence est conçue comme un défaut imputable à l'ignorance des causes nécessaires ${ }^{14}$. Elle n’a

[10] Comme on le sait, Hegel entend élever la philosophie à la dignité d'une science (G.W.F. Hegel, GW9, Phänomenologie, p. 11), dont le but est d'exprimer la nécessité intrinsèque de ce qui est. La vraie pensée est ainsi selon lui «pensée de la nécessité» (Hegel, TWA 8, Enzyklopädie, Frankfurt : Suhrkamp,1986, \$119 Z, p. 246). Chez Spinoza, la nécessité est ce en quoi sorigine l'ensemble de ce qui est. "Les choses n'ont pu être produites par Dieu d'aucune manière autre et dans aucun ordre autre, que de la manière et dans l'ordre où elles ont été produites. » B. SPINOzA, Ethique, trad. Appuhn, Euvres III, Paris : GF 1965, Livre I, Proposition XXXIII, p. 56.

[11] «Il n’est rien donné de contingent dans la nature, mais tout y est déterminé par la nécessité de la nature divine à exister et à produire quelque effet d'une certaine manière.» Ibid., Proposition XXIX, p. 52.

[12] Ces caricatures de la pensée de Hegel se basent sur une célèbre proposition de ses Principes de la philosophie du droit : «Was vernünftig ist, das ist wirklich; und was wirklich ist, das ist vernünftig.» Pour une interprétation informée de ce propos hégélien, on lira J-F. KERVÉGAN, Leffectif et le rationnel, Paris: Vrin 1997, pp. 17-32.

[13] Cf. HegeL, Wissenschaft der Logik, GW 11, pp. 369-409.

[14] «Pour nulle autre cause maintenant une chose n’est dite contingente, sinon eu égard à un manque de connaissance en nous ; car une chose dont nous ignorons que l'essence enveloppe contradiction, ou de laquelle nous savons bien quelle n'enveloppe aucune contradiction, sans pouvoir rien affirmer avec certitude de son existence, parce que nous ignorons l'ordre des causes, une telle chose, dis-je ne peut jamais nous apparaitre ni comme nécessaire ni comme impossible et, par suite, nous l'appelons contingente ou possible.» B. SpINOzA, Ethique, trad. Appuhn, op. 
pas de statut ontologique, son statut est seulement epistémique. Quand on est affecté par une idée dont on ne connaît pas la cause, on va imaginer que cette chose est contingente ou possible. La contingence est donc de l'ordre d'une fiction visant à combler une lacune de notre entendement. Il n'y a donc pas de nécessité de la contingence chez Spinoza, mais il y a sur le plan épistémique une contingence de la nécessité, car cette dernière peut nous échapper étant donné le mode fini de notre connaissance qui nous pousse à considérer une chose indépendamment de la totalité de ses causes.

Chez Hegel, la contingence est un moment à l'œuvre dans la nécessité effective. Le tout a besoin d'intégrer en son sein la contingence pour pouvoir être absolu. Préserver la nécessité du tout en rendant impossible la contingence aurait comme conséquence de relativiser ce tout par rapport à la contingence qui constituerait un extérieur qu'il ne pourrait assimiler. Plutôt que d'exclure la contingence, un tout qui se veut nécessaire et absolu, comme c'est le cas du système hégélien, se doit donc de l'inclure, d'en faire un moment de la nécessité totale.

Cela ne signifie toutefois pas que tout serait contingent, cela signifie plutôt qu'il y a de la contingence dans tout. Si Hegel fait de la nature le lieu privilégié de la contingence ${ }^{15}$, il reconnaît qu'elle est aussi à l'œuvre dans le monde de l'esprit. Cette impuissance qui rend les choses indéterminées et contingentes se retrouve au sein du monde de l'esprit. Hegel note ainsi dans l'introduction à son Encyclopédie : "qui serait assez sot pour ne pas observer autour de lui nombre de réalités qui ne sont pas en fait comme elles devraient être ?» ${ }^{16}$. La reconnaissance d'une contingence dans le monde ne signifie nullement pour Hegel qu'il faille en rester là. Les modalités aléthique n'expriment pas pour lui la fixité d'une situtation indépassable, mais des moments dans un procès d'effectuation du rationnel. Dans celui-ci, il y a une imbrication des modalités, qui fait qu'aucune n'est sans appel.

Hegel analyse cette co-implication des catégories modales dans le chapitre qu'il consacre à l'effectivité (Wirklichkeit) à la fin de la doctrine de l'essence ${ }^{17}$. L’originalité de Hegel dans ce chapitre tient au fait qu'il ne pense pas les différentes catégories modales comme des entités indépendantes. La possibilité, la contingence, l'impossibilité et la nécessité sont co-constitutives et forment une logique dynamique. En d'autres mots, loin d'appliquer les modalités à une

cit., Livre I, Proposition XXXIII, Scolie I, pp. 56-57.

[15] Si lon trouve des traces du rationnel dans la nature, celui-ci échoue à subsumer l'entièreté de celle-ci. Il y a en fait pour Hegel une «impuissance de la nature à tenir fermement les choses selon le concept». Hegel, Encyclopädie, GW 20, \$250A.

[16] HegeL, Encyclopädie, GW20, \$6A.

[17] Voir. Hegel, Wissenschaft der Logik, GW 11, pp. 380-392. 
réalité statique, Hegel essaye de montrer comment les catégories modales jouent un rôle dans l'effectivité d'un monde s'accomplissant. Il part alors du possible et montre comment, celui-ci se lie à l'effectivité en passant par le moyen-terme de la contingence et en s'accomplissant dans une nécessité interne.

Pour analyser les modalités aléthiques, Hegel part de la définition du possible comme non-contradiction. Mais la notion formelle du possible, qui est celle utilisée dans la logique classique, est, pour lui, insuffisante. Elle doit être nécessairement repensée si l'on veut comprendre le réel comme effectivité. Le possible formel, qui signifie que «tout est possible qui ne se contredise pas ${ }^{18}$ ", est une simple «abstraction" seulement relative à une "pensée subjective». $\mathrm{Si}$, d'un point de vue formel, la possibilité semble être impliquée dans la notion d'effectivité ${ }^{19}$, elle demeure, en même temps, différente de l'actualité. La signification de la possibilité est en relation avec quelque chose qui peut être actualisé, mais qui ne l'est pas encore. La possibilité est donc en relation avec quelque chose d'autre qu'elle-même. Dire que «ce qui est effectif est possible» n'est pas suffisant pour être définitoire, il faut ajouter que «tout ce qui est possible n'est pas en tant que tel actualise $\mathrm{e}^{20}$ ». La possibilité formelle doit être distinguée du résultat du procès à travers lequel la possibilité s'actualise. L'unité de la possibilité formelle et de l'effectivité est ainsi quelque chose de contradictoire. La possibilité formelle et l'effectivité ne peuvent exister sur le même plan et en même temps. Si la chose est effective, elle n'est plus seulement possible ; et si elle est dite possible, elle n'est pas encore effective. Mais rien dans le possible formel ne nous indique comment passer de l'idée d'une chose à sa réalisation.

Pour le possible formel, l'effectivité demeure quelque chose d'étranger. Hegel tente alors de forger un concept novateur de possibilité qui puisse rendre compte de la médiation qui du possible formel mène à l'effectivité. C'est ainsi qu'il forge le concept de possibilité réelle. Celle-ci est une possibilité concrète qui apparaît comme un moyen - et non seulement une condition restrictive de la réalisation de la chose. C'est une "condition ${ }^{21}$ " productive de l'effectivité, laquelle présuppose pour être là que l'ensemble de toutes ses conditions soit rassemblé.

Quand toutes les conditions d'une chose sont entièrement présentes, elle entre dans l'effectivité; la complétude des conditions est la totalité telle qu'elle se trouve

[18] Ibidem, p. 382.

[19] «Was wirklich ist, ist möglich». Ibidem, p. 381.

[20] Hegel, GW 20, \$ 143.

[21] Ibidem, $\$ 146$. 
dans le contenu et le quelque chose lui-même est ce contenu déterminé comme étant également effectif et possible ${ }^{22}$.

La somme exhaustive des conditions rassemblée, la possibilité réelle n'est plus la possibilité indifférente d'une effectivité. Elle est ce qui rend concrètement possible l'effectivité. Une fois qu'elle est comprise comme ce qui rend l'effectif vraiment possible, la possibilité prend la forme de la «manifestation» ou de la «nécessité réelle» ${ }^{23}$. Hegel parle ici de nécessité réelle dans la mesure où elle ne concerne pas seulement un mode de la connaissance, mais la caractérisation du procès d'effectivité du réel. La lexie de «nécessité réelle» est cependant ambiguë, car la nécessité réelle est tout d'abord réelle et formelle. Elle donne une forme nécessaire au contenu de la possibilité réelle, mais elle ne donne aucune nécessité à ce contenu en tant que tel. Elle n’est une nécessité réelle qu’en regard de la forme. En d'autres termes, la nécessité réelle, en rassemblant l'ensemble des conditions d'une effectivité exprime ce qui est nécessaire pour quelque chose, mais n'exprime en rien la nécessité de cette chose. La nécessité réelle présuppose en fait la possibilité réelle sans la poser ou la justifier. « Ce qui est vraiment nécessaire l'est en fait suivant sa forme, mais en regard du contenu, c'est quelque chose de limité, et qui de la sorte relève de la contingence ${ }^{24} . » \mathrm{La}$ nécessité ne couvre pas absolument son objet. C'est pourquoi, «la nécessité réelle est nécessité déterminée ${ }^{25}$ \%. En d'autres termes, c’est une nécessité qui est liée à la formation d'une effectivité, mais qui ne rend aucunement nécessaire le contenu de cette effectivité.

La nécessité réelle, qui est plus qu’une simple nécessité formelle, n’est pas encore la nécessité absolue qui est une nécessité déterminante. C'est seulement ce qui rend effectif une possibilité, mais pas ce qui la rend nécessaire. Pour Hegel, la nécessité absolue consiste à poser ses présuppositions. Elle n'actualise pas un contenu contingent, mais un contenu qui a été rationnellement décidé. La contingence n'est pas pour lui l'autre de la nécessité, mais un moment qui doit être dépassé si l'on veut penser l'effectivité dans son lien à un accomplissement de soi. Dans la nécessité absolue, le problème de la contingence du contenu est résolu dans la résolution réfléchie de se déterminer pour tel possible, dont les conditions sont disponibles. La nécessité absolue exprime ainsi la nécessité d'un contenu relativement à un agent libre et aux ressources dont il dispose. Elle relève de la logique de la liberté qui consiste à se réaliser dans ses productions en décidant de celles-ci parmi l'éventail du possible réel.

[22] Hegel, GW 11, p. 387.

[23] Ibidem, GW 11, p. 386.

[24] Ibidem, p. 389.

[25] Ibidem, p. 390. 
Contrairement à une façon de faire qui serait propre à un libre arbitre formel, il ne s'agit pas de se décider par un «libre décret ${ }^{26}$, de déterminer un idéal in abstracto et d'essayer de l'accomplir. Une telle façon de faire risquerait de nous conduire à un devoir être irréalisable. Il s'agit plutôt de lire le réel comme un champ de possibilités - qui une fois connectées - produisent du contingent ou du nécessaire pour peu que l'ordonnancement des possibles compris dans le réel soit pensé et posé par le sujet. En bref, il s’agit de voir ce qu’on peut faire avec ce qui est pour le faire être à sa plus haute puissance plutôt que d'essayer de faire quelque chose indépendamment de ce qui est.

Loin d'exclure le possible dans un mouvement linéaire de détermination, qui nous conduirait du possible au nécessaire en passant par le contingent, l'effectivité (Wirklichkeit) est un mouvement circulaire qui libère un possible pensé et voulu d'un contenu contingent. La nécessité hégélienne tient ainsi au fait de reconnaître, dans ce qui est simplement donné, la possibilité d'une réalisation du rationnel. La nécessité hégélienne est celle d'une libération, c'est une catégorie dynamique qui prédique un mouvement de réalisation et non la description d'une réalité qui vaudrait de toute éternité.

Dans cette perspective, la contingence ne définit pas, à l'instar de la tradition aristotélicienne, un type fixé de réalité, quelque chose qui est, mais qui tout aussi bien pourrait bien être ou ne pas être. La contingence est prise dans une perspective dynamique de réalisation, elle est, comme le commente Bernard Mabille, «l'inquiétude absolue de l'effectif qui se renverse en possible et du possible qui se renverse en effectif.» ${ }^{27}$.

Chez Spinoza, la contingence semble moins le moment d'une nécessité effective, le point de basculement du possible dans l'effectif ou de l'effectif dans le simple possible, qu'un point de vue ignorant la nécessité. Il y a ainsi, au niveau épistémologique, une «contingence de la nécessité» qui est fonction de la compréhension ou non que l'on a de la cause d'une chose. Pour Spinoza, nous considérons une chose comme contingente, si nous la considérons seule, sans avoir la moindre idée des causes qui pourraient la produire ${ }^{28}$; comme possible, si nous avons quelque idée de ces causes, mais sans savoir si elles entreront ou non en jeu pour la produire effectivement ${ }^{29}$; comme nécessaire,

[26] B. SpInoza, Oeuvres IV, op. cit., p. 304.

[27] B. Mabille, Hegel. L’épreuve de la contingence, Paris, Aubier, 1999, p. 209.

[28] «'appelle les choses singulières contingentes, en tant qu'ayant égard à leur seule essence, nous ne trouvons rien qui pose nécessairement leur existence ou l'exclue nécessairement.» B. Spinoza, Ethique, trad. Appuhn, Euvres III. Paris : GF 1964, Livre IV, définition 3, p. 221. «Si nous avons égard à l'essence d'une chose simplement mais non à sa cause, nous la dirons contingente». B. Spinoza, Pensées métaphysiques, trad. Appuhn, Euvres I. Paris : GF 1964, p. 347.

[29] «'appelle les mêmes choses singulières possibles, en tant qu'ayant égard aux causes 
si nous savons (ou croyons savoir) qu'elles agiront infailliblement. Du point de vue de la connaissance finie, la nécessité n'est pas nécessaire en tant que point de vue, mais contingente, car il y a chez Spinoza une certaine «impuissance de la raison ${ }^{30}$. Mais la converse ne se vérifie pas, il n’y a pas de nécessité de la contingence du point de vue de la nécessité. Chez Spinoza, les modalités ne peuvent pas être co-constitutives, car elles n'ont pas toutes le même statut. La nécessité et l'impossibilité ont un sens ontologique ; la possibilité et la contingence n'ont qu'un statut épistémique.

\section{LIMITES DE LA DOCTRINE SPINOZISTE}

En maintenant un ordre double, la théorie spinoziste trouve d'un point de vue hégélien son lieu logique dans la doctrine de l'essence ${ }^{31}$. Les modalités ne peuvent être co-constitutives ou imbriquées les unes dans les autres, car elles relèvent de deux statuts maintenus distincts. La nécessité et l'impossibilité ont une valeur ontologique alors que la possibilité et la contingence n'ont qu'une valeur cognitive. Une telle distinction est ce que Hegel entend pallier en pensant l'identité de l'être et de la pensée.

Généralement, pour ne pas relativiser la nécessité du système, on relativise la contingence et on en fait quelque chose de subjectif comme c'est le cas chez Spinoza, mais du coup on relativise aussi l'identité de la pensée et de l'être qui ne vaut plus dès lors que, par une certaine impuissance de la raison humaine, l'objet est perçu comme contingent. Cette dissociation de la pensée et de l'absolu signifie en fin de compte que la nécessité de l'absolu est indépendante de la pensée de cette nécessité. La pensée en s'élevant au point de vue de la nécessité ne fait que comprendre une nécessité déjà là. C'est pourquoi la pensée philosophique n'a pas besoin chez Spinoza d'un mode d'exposition propre, mais peut reprendre celui de la géométrie supposé exposer la nécessité de l'être.

Par ailleurs, la contingence objective de la nature n'est pas reconnue par Spinoza. La contingence n'a pas de statut ontologique. L'être des choses est nécessaire ou impossible. La contingence du monde est donc niée, c'est pourquoi Hegel parle d'un acosmisme chez Spinoza ${ }^{32}$. Loin de nier Dieu dans sa

par où elles doivent être produites, nous ne savons si ces causes sont déterminées de façon à les produire.» B. Spinoza, Ethique, op. cit., livre IV, définition 4, p. 221. «On dit qu'une chose est possible quand nous en connaissons la cause efficiente, mais que nous ignorons si cette cause est déterminée.» B. Spinoza, Pensées métaphysiques, trad. Appuhn, Euvres I. Paris : GF 1964, p. 347.

[30] Voir sur ce point : C. Lazzeri (éd.), Spinoza. Puissance et impuissance de la raison. Paris : Puf 1999.

[31] Hegel discute ainsi du système spinoziste dans sa Remarque au chapitre sur l'Absolu qui se trouve dans la Doctrine de l'essence.

[32] A titre d'exemple, on consultera à côté des Leçons sur la philosophie de la religion et 
conception d'un «Deus sive natura» ${ }^{33}$, ce que Spinoza aurait nié, ce serait la nature, le monde. La nécessité de la substance divine ne laisserait pas de place à la contingence ontologique du monde.

En bref, les deux reproches que l'on peut adresser à Spinoza d'un point de vue hégélien se formulent comme suit : 1) il n'aurait pas pensé l'identité de l'être et la pensée sous la forme d'une relation réciproque, mais sous la forme d'un déterminisme de la substance auquel la pensée doit se conformer (de façon géométrique $)^{34}$ et 2) il n'aurait pas attribué de statut susbistant à la contingence.

On peut toutefois se demander comment on peut faire droit à une nécessité absolue en accordant une objectivité subsistante à la contingence. Cela n'est possible que pour autant que l'on passe d'un cadre statique de pensée à un modèle dynamique. Pour éviter que la contingence ne relativise la nécessité, il faut la penser comme moment d'un procès constitutif du nécessaire. C'est ce que fait Hegel qui considère que les modalités relèvent de l'effectivité, c'est-à-dire de la correspondance processuelle du réel à la pensée. Cette correspondance est encore contingente dans le domaine de la nature, mais elle est rendue nécessaire pour ce qui relève du domaine de l'esprit s'accomplissant selon son concept.

Il n'y a pas, chez Hegel, d'un côté, des modalités objectives (le nécessaire et l'impossible) et, de l'autre côté, des modalités subjectives (le possible et le contingent), il y a un procès des modalités, dont le résultat, la nécessité absolue, est à la fois objectif et subjectif. Hegel prend le contrepied d'une tendance moderne qui, culminant avec Kant, lie les modalités au seul pouvoir de connaitre ${ }^{35}$. Chez Hegel, il y a une identité entre la pensée et le réel, mais celle-ci n’est pas d'emblée donnée comme une nécessité. La nécessité n'est pas absolue au sens où il n'y aurait qu'elle. Elle est absolue au sens où tout est nécessaire en elle, y compris la contingence.

sur l'histoire de la philosophie, la remarque au $\$ 50$ des Concepts préliminaires à l’Encyclopédie des sciences philosophiques. "Dans le système spinoziste, le monde est bien plutôt déterminé seulement comme un phénomène auquel ne saurait appartenir la réalité effective, de sorte que ce système est bien plutôt à regarder comme un acosmisme». Hegel, Encyclopédie (trad. Bourgeois). Paris : Vrin 1979, p. 313.

[33] B. Spinoza, Ethique, op. cit., livre 4, prop. 4, démonstration, p. 224.

[34] On notera que dans le texte introductif à sa théorie de la mesure, Hegel remarque qu’on pourrait attendre du mode spinoziste qu'il soit comme un troisième terme unifiant la substance à ses attributs. Mais le fait que ce troisième terme signe plutôt l'indifférence, le «sans [commune] mesure» indique bien à ses yeux que, seule, la substance compte.

[35] KanT, Critique de la raison pure. Paris : GF 2001, p. 277, A 219. 


\section{CONCLUSION}

Spinoza et Hegel ont en commun de faire de l'absolu le centre de gravité de leur système respectif. Mais ils se distinguent par la façon dont ils le concoivent. L'absolu de Spinoza ne dépend pas de la connaissance que l'on en a, il demeure nécessaire quand bien même notre ignorance nous pousserait à le poser comme seulement possible ou contingent.

Du point de vue d'une nécessité absolue, le contingent n'existe pas. C'est pourquoi, dans la mesure où l'homme aspire à ce point de vue, ce qui est contingent, l'affecte moins que ce qui est possible ou nécessaire ${ }^{36}$. Le problème du contingent est dès lors minimisé, il est ce qui a le moins de puissance. Si cela semble vrai d'un objet posé comme contingent, on peut toutefois douter que si a contingence est rapportée au sujet, elle l'affecte moins que la possibilité ou que la nécessité. A la contingence de l'être humain, à son caractère mortel, Spinoza affecte toutefois de ne pas accorder d'attention ${ }^{37}$. Sans aller jusqu'à renier la contingence de l'existence humaine, il pense que la liberté n'en relève pas. Il semble curieusement ignorer que la question de la mort fasse partie de la vie humaine. Si la contingence de l'existence qu'elle semble induire n'est due qu'à l'ignorance du point de vue divin, il faudrait alors expliquer la nécessité de la mort humaine de ce point de vue. Mais Spinoza n'en fait rien. Au lieu de ramener le problème de la contingence de l'existence à une ignorance due à la finitude de notre entendement, il asserte que quand on est libre, on ne s'occuppe que de la vie et non de la mort ${ }^{38}$. Ici, la nécessité n'est pas a transfiguration de la contingence dans un savoir des causes, mais le refoulement de celle-ci. Le refus de la contingence n'est pas toujours aussi explicite chez Spinoza, mais de façon générale, la libre nécessité, est indifférente au procès marqué par la contingence de l'être, qui y a conduit. Le problème est que, ce faisant, la liberté ne se rapporte qu'à elle-même ; elle n'est pas libération, car l'épreuve de son autre ne l'affecte pas vraiment.

[36] B. SpInoza, Ethique, livre 4, prop. XI-XIII.

[37] «L'homme libre ne pense à rien moins quà la mort, et sa sagesse est une méditation non de la mort, mais de la vie.» Ethique, prop. 67. Chez Hegel, l'importance de la mort est explicitement théorisée. Sur le sujet, voir : G. LeJeune, Hegel anthropologue, Paris: CNRS-éditions 2016, pp. 59-85..

[38] Comme on le voit le domaine gnoséologique est subordonné à une exigence éleuthériologique (Voir aussi B. Spinoza, Les principes de la philosophie de Descartes, in CEuvres, I, 273-274). On notera que l'ontologie, dans la mesure où lêtre procède de la nécessité divine qui, étant causa sui, est libre, relève également du domaine d'une éleuthériologie. A cet égard, le problème de Spinoza tient moins à un supposé substantialisme, qu'au fait de faire reposer lêtre et, partant, la connaissance sur la liberté sans pouvoir définir celle-ci autrement que formellement. 
En voulant intégrer le point de vue du sujet à la nécessité de la substance, Hegel entend penser une nécessité qui intègre comme moment (au sens dynamique de momentum) le procès cognitif marqué de contingence qui y conduit. A l'encontre de Spinoza, la nécessité chez Hegel ne se réaliserait dès lors que dans et par le contingent. La nécessité ne deviendrait présence à soi que pour autant qu'elle fasse retour sur soi ce qui présuppose un étrangement dans une altérité effective. La contingence, dans son rapport à la nécessité, acquiert dès lors un sens original chez Hegel, ce n'est ni une simple illusion, ni une facticité nécessaire, c'est l'espace dans lequel un système libre va se définir, c'est «le terrain nécessaire d'effectuation de la liberté» ${ }^{39}$.

Un lien entre nécessité et liberté existe certes déjà chez Spinoza, mais pour Hegel, il y est seulement à l'état d'intuition ${ }^{40}$, car il y manque une pensée de la liberté dans la contingence. Ce qui est en jeu dans la pensée de la nécessité spinoziste, c'est la libération de ce qui est subi comme autre. Il s'agit d'être chez soi dans l'autre. Le problème, pour Hegel, est que Spinoza n'aurait pas suffisamment reconnu cet autre qu'il s'agit de s'approprier, ne fut-ce que par la pensée. Il n'aurait pas accordé à la contingence une subsistance propre et aurait relativiser le monde par rapport à la toute puissance divine.

Si dans l'ordre épistémique, la contingence est reconnue comme la modalité d'un point de vue déficient, elle n'a aucun rôle constitutif dans l'éleuthériologie spinoziste tant celle-ci est occultée sous les dehors d'une ontologie substantialiste. Les illusions de l'imagination n'ont aucune valeur, elles présentent l'expression de la liberté comme celle d'un libre décret empêchent d'être véritablement libre, car, en substituant des fins arbitraires à la connaissance des causes, elles détournent d'une compréhension du tout qui, seule, pourrait nous permettre de nous réconcilier avec notre existence, de ne pas la subir comme un destin, mais d'y voir le fruit d'une libre nécessité ${ }^{41}$. Mais, en pensant avec Spinoza contre Spinoza, on dira que pour qu'il soit question d'une libre nécessité, il faut que celle-ci soit choisie contre la contingence, laquelle apparaitrait alors comme l'autre constitutif de la nécessité. La contingence serait dès lors un moment qui fait de la connaissance des causes nécessaire une sorte de libération. En bref, Spinoza aurait raison de ne pas faire reposer la liberté sur la contingence de l'arbitre, il aurait cependant tort de croire que la contingence n'est qu'une forme d'ignorance. En faisant de la contingence quelque chose qui ne constitue pas un moment de la nécessité, mais seulement un moment de notre accession à celle-ci, Spinoza dissocie le procès de la pensée de la nécessité de l'être, il fait de la substance et de ses modes des entités sans commune mesure. Chez lui, la

[39] B. Mabille, Hegel. Lépreuve de la contingence, op. cit., pp. 320-321.

[40] Hegel, GW 20, \$ 159.

[41] Voir B. Spinoza, Lettre à Schuller (1674), Oeuvres IV, op. cit., p. 304. 
nécessité de l'être, une fois comprise, annule d'un point de vue épistémique la contingence en tant qu'elle l'explique. Mais du point de vue de l'éleuthériologie hégélienne ${ }^{42}$, la contingence n'est pas annulée, car elle est ce par quoi libération il y a, ce par quoi expérience propre il y a. La nécessité du rationnel, en tant qu'elle concourt à la liberté, exclut moins la contingence qu'elle ne conclut un ordonnancement de celle-ci. La contingence est ainsi le moment par lequel toute nécessité comprise comme libération doit passer. Ce n'est qu'en ce sens que la philosophie s'accomplira comme "science de la liberté»" ${ }^{43}$ et fera de sa nécessité, celle d'une liberté se réalisant dans la contingence d'un monde trouvé.

\section{BiBLIOGRAPHIE}

F. H. Bradley, Appearance and Reality. London : Allen and Unwin $1897^{2}$.

M. Gueroult, Philosophie de l'histoire de la philosophie. Paris : Aubier-Montaigne 1979.

G.W. Hegel, Gesammelte Werke. Hamburg: Meiner 1968ss.

V. HösLE, «Begründungsfragen des objektiven Idealismus», in Philosophie und Begründung. Frankfurt: Suhrkamp, 1987.

I. Kant, Critique de la raison pure. Paris : GF 2001.

J-F. Kervégan, L'effectif et le rationnel. Hegel et l'esprit objectif. Paris : Vrin 2007.

J-F. KervéGan, B. Mabille (éds.), Hegel au présent. Une relève de la métaphysique? Paris : CNRS-éditions 2012.

A. KojÈve, Le concept, le temps et le discours. Introduction au système du savoir. Paris : NRF, 1990.

C. Lazzeri (éd.), Spinoza. Puissance et impuissance de la raison. Paris : Puf 1999.

G. Lejeune, Hegel anthropologue, Paris : CNRS-éditions 2016.

B. Mabille (éd.), Ce peu d'espace autour. Six essais sur la métaphysique et ses limites. Chatou : La Transparence 2010.

B. Mabille, Hegel. L'épreuve de la contingence. Paris : Aubier 1999.

[42] A côté de l’ontologie ou de lépistémologie, léleuthériologie, science de la liberté, définirait un point de vue original à travers lequel il importerait, comme le montre de façon convaincante Bernard Mabille, de lire Hegel et de cheminer avec lui (Voir B. Mabille, Hegel. Lépreuve de la contingence, op. cit., p. 53).

[43] Hegel, GW 13, Encyklopädie (1817), § 5. 
Q. Meillassoux, Après la finitude. Essai sur la nécessité de la contingence. Paris : Seuil, coll. L'ordre philosophique, 2006.

B. SpInoza, Euvres, traduction Appuhn, vols. I-IV. Paris : GF 1964.

Guillaume Lejeune is Collaborateur scientifique at the Universite Libre de Bruxelles and Chargé de recherches FNRS at the Université de Liège
AdDress:
Dr Guillaume LEJEUNE
Rue du Collège, 48
5530 Godinne

Belgique

EMAIL:

Guillaume.lejeune@ulg.ac.be

glejeune@ulb.ac.be

RESEARCH INTERESTS:

German Idealism, German Romanticism, British Idealism, Modern Philosophy.

Publications majeures :

G. Lejeune, Sens et usage du langage chez Hegel. Paris : Hermann 2014.

G. LEJEune (éd.), La question de la logique dans l'idéalisme allemand. Hildesheim: Olms 2013. 